\title{
A Novel Firefly Algorithm based on Improved Learning Mechanism
}

\author{
Qiang $\mathrm{Fu}^{1,2}$, Zheng Liu ${ }^{1}$, Nan Tong ${ }^{1}$, Mingbo Wang ${ }^{1}$, Yiming Zhao ${ }^{1}$ \\ 1College of Science and Technology, Ningbo University, China \\ No.505, YuXiu Road, Ningbo 315212,China \\ 2 Faculty of Information Science and Engineering, Ningbo University, Ningbo, China \\ No.808, FengHua Road, Ningbo 315211, China \\ Corresponding author, e-mail: fuqiang@nbu.edu.cn
}

\begin{abstract}
According to the problem of low solution precision, slow convergence speed and some fireflies failure in the traditional firefly algorithm, an improved firefly optimization algorithm is proposed based on the learning mechanism, By chaotic maps, the Firefly's initial position in which dynamic distance weighting is introduced to strength the search ability of algorithm, and adaptive-step scheme is used to balance the dual requirement of local and global optimization. Gaussian mutation is also adopted in the algorithm to help firefly population jump out of local optimum effectively. Experimental results show that the proposed algorithm has rationality and effectiveness.
\end{abstract}

Keywords-Firefly Algorithm; Distance Weighting; Chaos; Gaussian Mutation; Learning Mechanism

\section{INTRODUCTION}

The swarm intelligence refers to the intelligent behavior showed by the subject having no wisdom or having simple wisdom through the cooperation and communication. It provides a new idea for finding and solving the complicated distributed problem on the premise of having no centralized control and failing to provide the global model. Currently, the most common swarm intelligence algorithms mainly include: bee colony algorithm, ant colony algorithm, particle swarm optimization, fish swarm algorithm and the like. These intelligence algorithms have been widely used in many fields due to their many advantages.

The firefly algorithm (FA) was proposed by Xin-She Yang, professor of University of Cambridge in 2008, which was called as FA [1]. This algorithm is enlightened by the firefly for passing information through brightening at night, and is a new swarm intelligence optimization algorithm based on the population search. The proposal time of this algorithm is relatively short, but the parameters needing to be adjusted during the operation are relatively few due to its simple structure; and the optimizing search capability is also relatively good, therefore, it is widely used in the fields, including software testing [2], combinatorial problem [3], network route selection [4], price budget [5], automatic control system optimization [6] [7] and so on. Currently, the optimization studies the firefly algorithm performance at abroad is still in the primary stage, but the improvement study carried out by many scholars on it has a certain achievement. For example, the chaos optimization algorithm is combined in [8] and improved algorithm proposed by Gandomi [9] at abroad. The thoughts of these algorithms are to convert the solution space from chaos space to the solution spaces, and then, to search optimization with the randomness and ergodicity of the chaos variable, which can help to jump out the minimum point locally. But, the current chaos optimization algorithm adopts the uniform mutation operation when mutating, so that the improved firefly algorithm cannot find the globally optimal solution rapidly in the limited iterations, and a big randomness is provided in the solving process, thereby, increasing the calculating amount of the algorithm. Fu[10] indicated that the algorithm was not converged easily through a multi-population mechanism, and the defect of the low solving accuracy was indeed improved to some extent. However, when many 
sub-populations are converged together, it will fall into the local optimum at the same time, causing that the learning mechanism of the multi-population is failed, and falling into the locally optimal solution. It is worthy of further studying how to strengthen the population diversity to improve the optimizing accuracy at the same time of positioning the optimum solution area rapidly.

In view of the disadvantages of the original firefly algorithm and currently improved algorithm in the function optimization, this paper proposed a Normalization Firefly Algorithm (NFA) based on the improved learning mechanism by studying the evolution mechanism of the firefly, and finally, verified the effectiveness of the improvement scheme proposed in this paper by 6 standard testing functions.

\section{Basic Firefly Algorithm (FA)}

\section{A. Bionics principle of algorithm}

In nature, the firefly will emit the short and rhythmed fluorescence, and the implications of the light emitted by different kinds of the fireflies are different. FA is a random optimization algorithm built by simulating the group behavior of the firefly, but some original biological significances of the firefly luminescence are omitted; and researchers search our companies in the feasible region only with their luminescence characteristics in the algorithm, and move towards the brightest fireflies in our vision field, thereby, realizing the updating of our locations.

In the algorithm, the fluorescent brightness and attraction degree of the firefly are the two critical factors in the algorithm process. The fluorescent brightness decides the moving direction and attraction power of the firefly; if the brightness is higher, it shows that the current target value is better, the brighter firefly will have the stronger attraction and can attract the firefly of which the brightness is less bright than it within the scope that the sight can bear. If there are not fireflies which are brighter than them, the fireflies will fly randomly in their fields. The optimization of the target is achieved by continuously updating the attraction degree and fluorescent brightness.

\section{B. Mathematical description and analysis}

Based on the above statement, the optimizing principle of FA is described as follows from the angle of the mathematical implication:
Definition 1 The relative fluorescent brightness of the firefly can be defined as:

$$
I=I_{0 i} \times e^{-\gamma r_{i j}}
$$

Wherein, $I_{0 i}$ indicates the fluorescent brightness of the $\boldsymbol{i}$-th firefly, and also the target function value corresponding to the $\boldsymbol{i}$-th firefly; and if the brightness of the firefly is higher, it shows that the target function value is more optimum. $\gamma$ indicates the absorption factor of the luminous intensity, which shows the characteristic that the fluorescence is gradually changed due to the influence of the communication media and distance. Generally, the value is set as the constant; and $r_{i j}=\left\|x_{j}-x_{i}\right\|$ indicates the spatial distance between the $\boldsymbol{i}$-th firefly and the $\mathrm{j}$-th firefly.

Definition 2 The attraction degree of the firefly can be defined as:

$$
\beta=\beta_{\circ} \times e^{-\gamma r^{2} i}
$$

Wherein: $\beta_{0}$ indicates the maximum attraction degree, namely, the attraction degree of the maximum fluorescent brightness; and in Formula (2), it describes the characteristic that the attraction of the fluorescence emitted by the firefly to other individuals is gradually reduced as the attraction and distance of the communication media are increased.

Definition 3 The evolution of the position that the j-the firefly attracts the $i$-th firefly in the process of optimization is expressed with Formula (3):

$$
x_{i}=x_{i}+\beta\left(x_{j}-x_{i}\right)+\alpha(\text { rand }-1 / 2)
$$

Wherein: $x_{i}, x_{j}$ indicate the spatial position located by the $\boldsymbol{i}$-th firefly and the $\mathrm{j}$-th firefly; $\alpha$ indicates the step size, and $\alpha \in[0,1]$; and rand indicates the random factor uniformly distributed on $[0,1]$. The search scope of the firefly can be increased by adding disturbing term $\alpha \times($ rand $-1 / 2)$ in the location updating process, thereby, improving the optimization capacity, and 
avoiding the early convergence of the firefly; and the position optimization of the firefly can be realized upon Formula (3). The location updating is carried out on the firefly population for many times, and finally, all fireflies gather near the firefly having the stronger fluorescence, thereby, realizing the optimization of the algorithm.

\section{A novel Firefly Algorithm (NFA) based on the improved learning mechanism}

In the traditional firefly algorithm, the initial position of the firefly is randomly produced in the search space, which may cause the non-uniform location distribution to greatly reduce the diversity of the algorithm. In the location updating process of the firefly, the attraction degree $\beta$ and step size $\alpha$ decide the direction of the evolution and final optimization result. But, the firefly algorithm has the following problems in the actual application: firstly, when the search domain is relatively big, the firefly individuals having a big distance cannot exchange and learn, which causes that the optimization effect is not ideal; and secondly, the algorithm has good optimization capacity in the early stage of the iteration, but when it reaches the region near the locally optimal solution, the slow evolution is easily presented, and even the phenomenon of stagnation, at this time, the firefly population will surround near the locally optimal solution to vibrate, so it cannot converge to the optimal solution better, thus, causing the convergence precision is low, and the optimization result is not ideal. In view of the above-mentioned problems, this paper proposed the following several improvement schemes upon the evolution mechanism of the algorithm.

\section{A. Position initialization of the firefly based on the chaos sequence}

The chaos concept, as an emerging optimization method, is widely concerned in the engineering circle and academic circles, and used in the engineering fields.

The chaos is characterized with randomness, ergodicity, boundedness, regularity and the like. It is especially sensitive to the initial value, and can traverse all states in the given scope upon its own rules. In this paper, it just uses the characteristic of the chaos to initialize the position of the firefly population, so as to lay the foundation for further improving the diversity of the global search.
The expression of cubic mapping is showed as follow:

$$
\begin{aligned}
& y(n+1)=4 y(n)^{3}-3 y(n) \quad-1 \leq y(n) \leq 1 \\
& n=0,1,2, \ldots .
\end{aligned}
$$

Firstly, D-dimensional spatial vector is produced randomly, namely, $Y_{1}=\left(y_{1}, y_{2}, \ldots y_{d}\right), \quad$ wherein $y_{i} \in[-1,1], 1 \leq i \leq D ; Y_{1}$ is used as the initial vector of the chaos iteration, and then, $\mathbf{M}$ chaos variables are produced upon Formula (4). The produced chaos variable is mapped to the various dimensional value spaces of the optimization variable upon Formula (5):

$$
x_{i d}=L_{d}+\left(1+y_{i d}\right) \frac{U_{d}-L_{d}}{2}
$$

Wherein: $L_{d}$ and $U_{d}$ indicate the lower limit and upper limit of the search space on the d-th dimension, $y_{i d}$ indicates the d-th dimension of the $i_{\text {-th spatial vector }}$ produced by Formula (4), and $x_{i d}$ indicates the coordinate of the d-th dimension of the $i_{\text {-th firefly in the }}$ search space. Then, the target function value is calculated upon the obtained position coordinates, the obtained results are sorted, and $\mathrm{n}$ individuals having the optimal target value are selected as the initial population.

\section{B. Dynamic distance weighting mechanism}

By analysis on the optimization mechanism of the FA, it can be known that, the learning and exchange between the fireflies must be within the visual range. If the firefly is relatively few and the range is relatively big, it will be hard for the firefly having a far distance to get the information of other fireflies, and can swing at the initial position, thereby, hindering the information exchange between the individuals.

In view of the above causes, it can be seen from the definition of the attraction degree of the firefly in Formula (2) that, the attraction degree $\beta$ is related to the distance $r_{i j}$.

This paper defines the exponential part of the spatial distance as the distance weight, which is expressed with $\omega$. After the test comparison of many different $\omega$ values, it is found that when $\omega$ is relatively big, the attraction power of the firefly will be limited in a small 
range, and the firefly can also search locally, which causes that the algorithm easily falls into the local optimum; otherwise, when the value of $\omega$ is relatively small, the attraction range of the firefly will be relatively big, at this time, the firefly can search in a big range, and has the global search capacity. In the traditional firefly algorithm, the distance weight is a constant value, which greatly limits the optimization capacity of the algorithm, so the study on the distance weigh seems more important.

To sum up, this paper proposed that the dynamic distance weighting mechanism was showed in Formula (6):

$$
\omega(i)=\omega_{\max }-\left(\omega_{\max }-\omega_{\min }\right)\left(\frac{i}{G}\right)^{2}
$$

Wherein: $\omega_{\max }$ indicates the initial distance weight value of the, and generally, $\omega_{\max } \in[2,4] ; \omega_{\min }$ indicates the distance weight value when iterating to the maximum times, and generally, $\omega_{\min } \in[0.5,1] ; \mathrm{G}$ indicates the maximum iterations; and $i$ indicates the current iterations. By setting in this way, the amplitude variation of $\boldsymbol{\omega}$ is small, so, it is unlikely to fall into the local optimum fast.

\section{Adaptive step mechanism}

The value of the step size $\alpha$ affects the convergence of the algorithm. According to the experiment, it is known that the local optimal point is easy to be reached when the value of the step size $\alpha$ is large relatively, and therefore the task of global search can be realized well; however, this point cannot be converged easily in the late period of algorithm running, and the phenomenon of oscillation is easy to be presented. Moreover, the algorithm is easy to be caught in local optimum when the convergence precision is improved as the value of the step size $\alpha$ is small. Finally, the optimization results are unsatisfactory.

Based on this idea, researchers introduced a decline exponent $\lambda$ and an iteration threshold $G_{0}$, and proposed an adaptive and variable step mechanism as shown in Formula (7):

$$
\alpha(i)=\alpha_{\max }-\left(\frac{i-1}{G_{0}-1}\right)^{\lambda}\left(\alpha_{\max }-\alpha_{\min }\right)
$$

Wherein, $\alpha(i)$ indicates to the step value of the generation $i, \alpha_{\max }$ indicates the initial and maximum step size; $i$ indicates the current iteration number, $\lambda$ indicates the decline exponent and it shows declining rate. When the iteration number is $G_{0}$, realize $\alpha(\mathrm{i})=\alpha_{\min }$ and keep that until the completion of the search. A relatively large step size $\alpha$, set in the early stage of the algorithm running, is conducive to the completion of global search. With the implementation of iteration, the step size $\alpha$ is in nonlinear reduction, wherein the reduction rate is slow, which ensures that the algorithm will not be caught in local optimum prematurely. When the iteration threshold $G_{0}$ is reached in the late iteration period, the step size is $\alpha_{\min }$ constantly, which is conducive to the improvement of convergence precision of the algorithm when the global optimum is searched in the late period of the algorithm.

\section{Gauss mutation mechanism}

If the population is caught in an optimal value in the process of algorithm running, and the condition of local optimum or global optimum cannot be determined, researchers will implement disturbance to the fireflies with gauss mutation factor. Gaussian distribution, which is also known as normal distribution, is a probability distribution widely applied in mathematics and engineering, wherein the expression of probability density function is shown in Formula (8):

$$
f(x)=\frac{1}{\sqrt{2 \pi} \sigma} e^{-\frac{(x-\mu)^{2}}{2 \sigma^{2}}}
$$

In the Formula, $x \in(-\infty,+\infty)$ and $\mu$ indicate expected values, $\sigma$ indicates variance value.

For the optimal solution of each iteration of the population, the variance for the optimal solutions of the continuous $\mathrm{S}(\mathrm{S}<\mathrm{M})$ time(s) will be calculated when the iteration number is more than $\mathbf{M}(\mathbf{M}>30)$, wherein it can be indicated that the optimal solution of the population has been searched and the algorithm cannot be ejected therefrom when the variance value is less than a certain precision $\eta$. Now, researchers will subject the fireflies in the currently local optimum position into gaussian mutation, and the Formula thereof is shown in Formula (9):

$$
x_{\text {best }}=x_{\text {best }}+x_{\text {best }} * N(\mu, \sigma)
$$

In the formula, $x_{b e s t}$ indicates the position of the currently optimal solution, and $N(\mu, \sigma)$ indicates the compliance the gaussian distribution based on the 
variance $\sigma$ and the expected value $\mu$.

Due to the self adjustment on the population of fireflies, the population can be ejected from local optimum, and the global search ability of the population can be improved.

\section{E. Flow of NFA algorithm}

In conclusion, the flow of NFA algorithm is as follows:

Step 1 Initialize the basic parameters of the algorithm, and then set the fireflies number $\mathrm{n}$, strong light absorption coefficient $\gamma$, the maximum attract degree $\beta_{0}$, the maximum iteration threshold $G_{0}$, the step size, range weight, the maximum iteration number Max Generation, and the decline exponent $\lambda$.

Step 2 Determine the initial population according to the fireflies location initialization method based on the chaos sequence mentioned above, and take the corresponding objective function value of each firefly as their biggest fluorescence intensity $I_{。}$.

Step 3 Calculate the relative brightness I of fireflies in the group according to Formula (1), and then sort the fireflies in the whole field according to the size of the fluorescence intensity.

Step 4 Alternate the range weight and shrinkage step according to Formula (6) and (7).

Step 5 Calculate the attraction degree $\beta$ of fireflies group according to Formula (2), and then determine the evolution direction of fireflies by combining with the relative brightness in the third step.

Step 6 Update the spatial location of fireflies according to Formula (3), and then calculate the brightness of the fireflies again according to the updated location of fireflies.

Step 7 Determine whether the population iteration number has reached to $\mathrm{M}$ time(s) and whether the variance for the optimal solution of continuous $\mathrm{S}$ time(s) is less than, then implement gauss mutation according to Formula (9) when the conditions mentioned above are met; otherwise, turn to step 8.

Step 8 Determine whether the iteration number has reached to the iteration threshold $G_{0}$, then change the step size to $\alpha_{\min }$ when the condition mentioned above is met; otherwise, alternate the range weight according to Formula (6), and shorten the step according to Formula (7).

Step 9 Turn to step 10 while reaching to the maximum number of search; otherwise, increase the number of search by 1 , turn to step 3 to implement the search of next time.

Step 10 Output the global optimal solution after the completion of iteration.

The time complexity of the algorithm is $O\left(n^{2}\right)$, in which $\mathrm{n}$ indicates the number of fireflies.

\section{Experiment simulation and analysis}

This text adopts the six standard test functions to verify the convergence speed and optimization ability of NFA algorithm; and the six test functions are as follows:

$$
\begin{aligned}
& F_{1}(\mathrm{x})=20\left(1-e^{-0.2 \sqrt{0.5\left(x^{2}+y^{2}\right)}}\right)-e^{0.5(\cos (2 \pi x)+\cos (2 \pi y))}+e \\
& F_{2}(\mathrm{x})=-e^{-(x-4)^{2}-(y-4)^{2}}-e^{-(x+4)^{2}-(y+4)^{2}}-2 e^{-x^{2}-(y+4)^{2}}-2 e^{-x^{2}-y^{2}} \\
& F_{3}(x)=0.5+\frac{\left(\sin \sqrt{x^{2}+y^{2}}\right)^{2}-0.5}{\left(1+0.001\left(x^{2}+y^{2}\right)\right)^{2}} \\
& F_{4}(x)=1+\frac{1}{4000} \sum_{i=1}^{n} x_{i}^{2}-\prod_{i=1}^{n} \cos \left(\frac{x_{i}}{\sqrt{i}}\right) \\
& F_{5}(x)=\sum_{i=1}^{n-1}\left[100\left(x_{i+1}-x_{i}^{2}\right)^{2}+\left(1-x_{i}\right)^{2}\right] \\
& F_{6}(\mathrm{x})=\sum_{i=1}^{n} x_{i}^{2}
\end{aligned}
$$

The specific parameter setting of each test function is as shown in Table 1:

TABLE I. Parameter value of standard test function

\begin{tabular}{cccc}
\hline $\begin{array}{c}\text { Func } \\
\text { tion }\end{array}$ & $\mathrm{D}$ & Range & $\begin{array}{c}\text { Optimu } \\
\mathrm{m}\end{array}$ \\
\hline$F_{1}(\mathrm{x})$ & 2 & $(-10,10)^{N}$ & 0 \\
$F_{2}(\mathrm{x})$ & 2 & $(-10,10)^{N}$ & -2 \\
$F_{3}(\mathrm{x})$ & 2 & $(-10,10)^{N}$ & 0 \\
$F_{4}(\mathrm{x})$ & 10 & $(-100,100)^{N}$ & 0 \\
& 10 & $(-30,30)^{N}$ & 0 \\
$F_{5}(\mathrm{x})$ & 10 & $(-100,100)^{N}$ & 0 \\
$F_{6}(\mathrm{x})$ & & & \\
& & & \\
\hline
\end{tabular}


Firstly, in order to verify the optimization performance of NFA algorithm, researchers compare the optimization results of the improved Firefly algorithm (NFA) in the text by Firefly algorithm (FA), artificial firefly algorithm (GSO) and particle swarm optimization (PSO).

The population size $\mathrm{n}$ of each algorithm is set to 45 , the largest number of iterations Max Generation is 300.The rest parameter settings are as follows:

NFA algorithm: light absorption strength coefficient $\gamma=1$; maximum attraction degree $\beta_{0}=1$, initial distance weight $\omega_{\max }=2$, minimum weight $\omega_{\text {min }}=0.5$, iterative threshold $G_{0}=200$, initial step length $\alpha_{\max }=0.4$, minimum step length $\alpha_{\min }$ is $3 \times 10^{-4}$; and the decline exponent is $\lambda=2$.

Firefly algorithm (FA): light absorption strength coefficient $\gamma=1$; maximum attraction degree $\beta_{0}=1$, step length is 0.2 ; and the distance weight is 2 .

Artificial fireflies swarm algorithm (GSO): fluorescein volatile coefficient $\rho=0.4$, fluorescein update rate $\gamma=0.6, \beta=0.08, n_{t}=5$, initial fluorescein $l_{\circ}=5$, perception range $r_{\circ}=10$; and the initial step length $\mathrm{S}=0.03$.

Particle swarm optimization (PSO): inertia weight $\omega=0.5$, learning factor $C_{1}=C_{2}=2$

Table 2 is the statistical result for 20 times of independent tests of each algorithm.

TABLE II. comparison of test results of NFA with FA, GSO, PSO

\begin{tabular}{|c|c|c|c|c|c|}
\hline $\begin{array}{l}\text { Functio } \\
\mathrm{n}\end{array}$ & $\begin{array}{l}\text { Algorit } \\
\text { hm }\end{array}$ & Worst & Best & Mean & Std \\
\hline \multirow{4}{*}{$F_{1}$} & FA & $7.21 \mathrm{e}-02$ & $3.25 \mathrm{e}-03$ & $5.46 \mathrm{e}-03$ & $6.14 \mathrm{e}-05$ \\
\hline & GSO & $8.65 \mathrm{e}-03$ & $2.81 \mathrm{e}-03$ & $5.96 \mathrm{e}-03$ & $3.85 \mathrm{e}-06$ \\
\hline & PSO & 20.00023 & $9.23 \mathrm{e}-11$ & 12.01 & $1.06 \mathrm{e}+02$ \\
\hline & NFA & $5.12 \mathrm{e}-19$ & 0 & $7.17 \mathrm{e}-20$ & $2.27 \mathrm{e}-23$ \\
\hline \multirow{4}{*}{$F_{2}$} & FA & -1.97872 & -1.99993 & -1.99856 & $8.45 \mathrm{e}-03$ \\
\hline & GSO & -1.97956 & -1.99996 & -1.99890 & $6.91 \mathrm{e}-03$ \\
\hline & PSO & -1.94588 & -2 & -1.99278 & $1.53 \mathrm{e}-03$ \\
\hline & NFA & -2 & -2 & -2 & 0 \\
\hline \multirow{2}{*}{$F_{3}$} & FA & $8.12 \mathrm{e}-03$ & $3.51 \mathrm{e}-06$ & $5.71 \mathrm{e}-03$ & $4.08 \mathrm{e}-06$ \\
\hline & GSO & $3.72 \mathrm{e}-06$ & $4.80 \mathrm{e}-08$ & $2.54 \mathrm{e}-06$ & $1.93 e-12$ \\
\hline
\end{tabular}

\begin{tabular}{|c|c|c|c|c|c|}
\hline & PSO & $9.71 \mathrm{e}-03$ & $4.05 \mathrm{e}-11$ & $3.94 \mathrm{e}-03$ & $2.47 \mathrm{e}-05$ \\
\hline & NFA & 0 & 0 & 0 & 0 \\
\hline \multirow{4}{*}{$F_{4}$} & FA & 1.00253 & 0.46173 & 0.61855 & $6.16 \mathrm{e}-02$ \\
\hline & GSO & 0.88462 & 0.31648 & 0.61867 & $8.90 \mathrm{e}-03$ \\
\hline & PSO & $4.94 \mathrm{e}-06$ & $9.82 \mathrm{e}-10$ & $8.16 \mathrm{e}-07$ & $9.16 \mathrm{e}-12$ \\
\hline & NFA & 0 & 0 & 0 & 0 \\
\hline \multirow{4}{*}{$F_{5}$} & FA & $6.43 \mathrm{e}+04$ & $2.27 \mathrm{e}+02$ & $4.23 \mathrm{e}+03$ & $4.71 \mathrm{e}+09$ \\
\hline & GSO & $1.37 \mathrm{e}+04$ & $4.77 \mathrm{e}+02$ & $8.61 \mathrm{e}+03$ & $2.90 \mathrm{e}+09$ \\
\hline & PSO & 170.0451 & 9.0863 & 59.2452 & 51.9034 \\
\hline & NFA & 4.00000 & 3.99039 & 3.998765 & $8.54 \mathrm{e}-6$ \\
\hline \multirow{4}{*}{$F_{6}$} & FA & $2.03 e+02$ & 19.0734 & $4.65 \mathrm{e}+01$ & $1.02 \mathrm{e}+03$ \\
\hline & GSO & 107.7195 & 21.0404 & 49.6537 & $8.18 \mathrm{e}+02$ \\
\hline & PSO & $3.54 \mathrm{e}-09$ & $6.54 \mathrm{e}-11$ & $6.64 \mathrm{e}-10$ & $2.01 \mathrm{e}-09$ \\
\hline & NFA & $3.44 \mathrm{e}-255$ & 0 & $2.46 \mathrm{e}-246$ & 0 \\
\hline
\end{tabular}

It can be seen from Table 2 that the worst solution, optimal solution, average, variance and the like NFA algorithm are better than other basic algorithms in aspects of $F_{1}(\mathrm{x}), F_{2}(\mathrm{x}), F_{3}(\mathrm{x})$ two dimensional function solve and $F_{4}(\mathrm{x}), F_{5}(\mathrm{x}), F_{6}(\mathrm{x})$ high dimensional function solve compared with FA algorithm, GSO algorithm and PSO algorithm; and it shows stronger ability of optimization. Combined with variance, it can be seen that the improved algorithm has stronger robustness and adaptability.

Secondly, in order to further understand the NFA algorithm adaptability, researchers shall compare the evolutionary process of optimal adaptation degree of FA algorithm, GSO algorithm and PSO algorithm.

Figures 1-6 are NFA and FA, GSO, PSO find the optimal solution of the function value with the number of iterations. To facilitate the contrast observation, fitness value is a value every 10 .

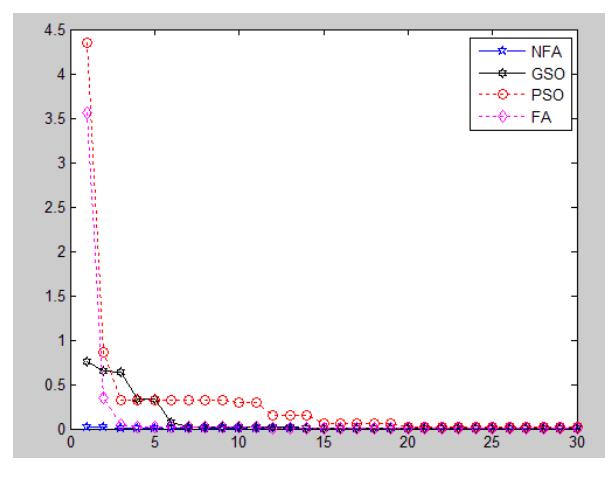

Figure 1. evolutionary fitness curve of F1 function 


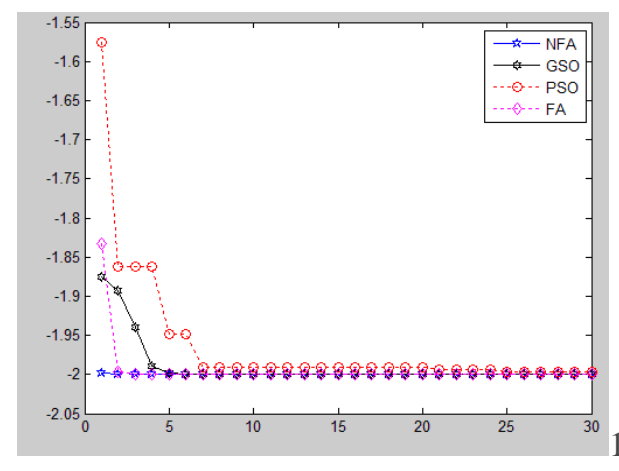

Figure 2. evolutionary fitness curve of F2 function

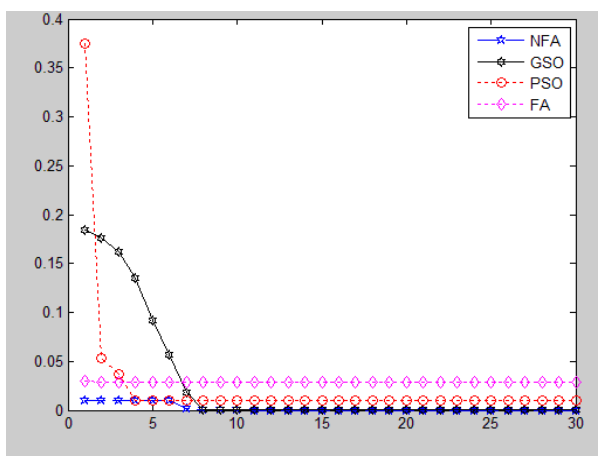

Figure 3. evolutionary fitness curve of F3 function

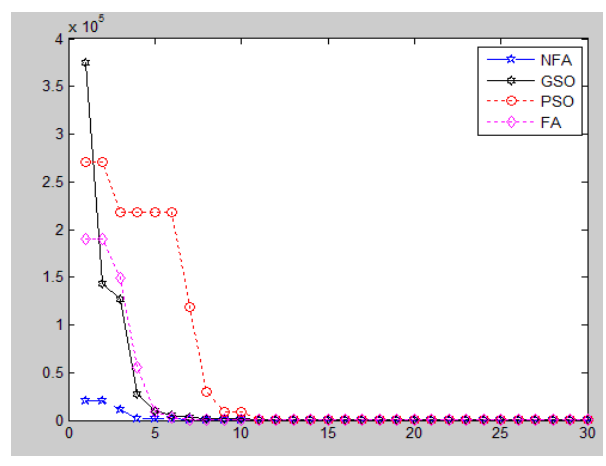

Figure 4. evolutionary fitness curve of F4 function

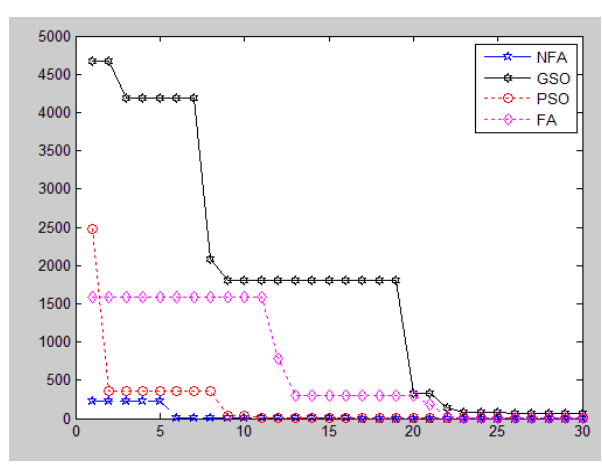

Figure 5. evolutionary fitness curve of F5 function

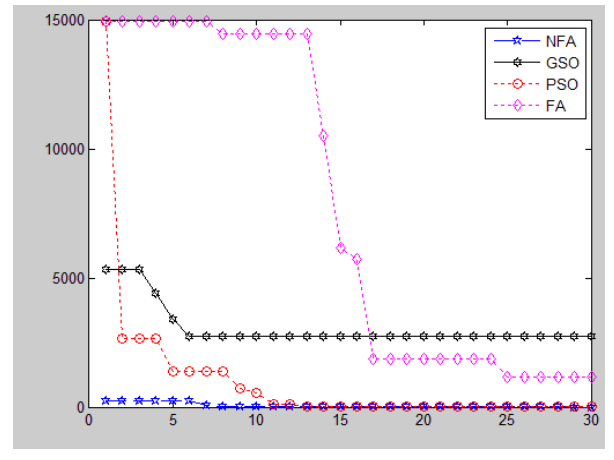

Figure 6. evolutionary fitness curve of F6 function

It can be seen from figures 1-6 that NFA algorithm has fast convergence rate and more stable operation under the same number of iterations. The improved algorithm of chaotic sequence is used to position population initialization, laid the foundation for global searching algorithm, convergence rapidly enough help, in the dynamic distance weighting mechanism driven by combining adaptive step length, the NFA algorithm can converge to the optimal solution faster near and precision were significantly higher than the other algorithms so as to better meet the local to the global search optimization; solving efficiency also has improved greatly.

Finally, in order to validate the effectiveness of the NFA Algorithm, the text is compared with Multi-Group Firefly Algorithm, MFA[13] and Chaos-based Dynamic Population Firefly Algorithm, CDPFA[11]; and Table 3 is the testing results of 20 independent tests under 45 population size and 300 iterations.

TABLE III. comparison of test result of NFA, MFA and CDPFA

\begin{tabular}{cccccc}
\hline Function & Algorithm & Best & Mean & Best & Time(s) \\
\hline \multirow{2}{*}{$F_{1}$} & NFA & $\mathbf{0}$ & $\mathbf{7 . 1 7 e - 2 0}$ & $\mathbf{2 . 2 7 e - 2 3}$ & $\mathbf{0 . 5 5 1 4 6 5}$ \\
& MFA & 0 & $8.87 \mathrm{e}-16$ & $2.49 \mathrm{e}-18$ & 2.427722 \\
& CDPFA & 0 & $8.88 \mathrm{e}-16$ & $5.21 \mathrm{e}-21$ & 1.384929 \\
$F_{2}$ & NFA & $\mathbf{- 2}$ & $\mathbf{- 2}$ & $\mathbf{0}$ & $\mathbf{0 . 4 6 8 1 8 6}$ \\
& MFA & -2 & -2 & $3.21 \mathrm{e}-294$ & 2.089253 \\
& CDPFA & -2 & -2 & $4.51 \mathrm{e}-307$ & 1.021872 \\
$F_{3}$ & NFA & $\mathbf{0}$ & $\mathbf{0}$ & $\mathbf{0}$ & $\mathbf{0 . 6 6 4 0 1 8}$ \\
& MFA & 0 & $5.78 \mathrm{e}-271$ & $3.56 \mathrm{e}-353$ & 2.281818 \\
& CDPFA & 0 & $4.18 \mathrm{e}-194$ & $7.14 \mathrm{e}-295$ & 1.547165 \\
& NFA & $\mathbf{0}$ & $\mathbf{0}$ & $\mathbf{0}$ & $\mathbf{0 . 3 7 3 7 0 2}$ \\
& MFA & 0 & $8.14 \mathrm{e}-18$ & $6.24 \mathrm{e}-22$ & 2.547014 \\
& CDPFA & $3.4 \mathrm{e}-304$ & $4.22 \mathrm{e}-12$ & $1.35 \mathrm{e}-17$ & 1.487345 \\
\hline & & & & &
\end{tabular}




\begin{tabular}{cccccc}
\hline & NFA & $\mathbf{3 . 9 9 0 3 9}$ & $\mathbf{3 . 9 9 8 7 6 5}$ & $\mathbf{8 . 5 4 e - 6}$ & $\mathbf{0 . 3 6 2 8 3 2}$ \\
$F_{5}$ & MFA & 8.92554 & 9.1054 & 1.24752 & 2.205257 \\
& CDPFA & 8.95789 & 9.69769 & 2.04164 & 1.302629 \\
& NFA & $\mathbf{0}$ & $\mathbf{2 . 4 6 e - 2 4 6}$ & $\mathbf{0}$ & $\mathbf{0 . 7 9 4 6 0 9}$ \\
\multirow{2}{*}{$F_{6}$} & MFA & $4.45 \mathrm{e}-235$ & $6.12 \mathrm{e}-106$ & $4.21 \mathrm{e}-210$ & 1.808695 \\
& CDPFA & $2.61 \mathrm{e}-166$ & $3.40 \mathrm{e}-101$ & $1.26 \mathrm{e}-200$ & 1.134946 \\
\hline
\end{tabular}

It can be seen from Table 3 that NFA algorithm and CDPFA algorithm and MFA algorithm can seek for the optimal solution during 20 tests accurately in low dimensional function optimization; the optimization rate of NFA algorithm of Ackley function reaches 98\%; CDPFA algorithm and MFA algorithm optimization rate reached $91 \%$ and $98 \%$, only Shaffer functions and Forepeaks NFA algorithm optimization rate reached 100\% and is very sTable and CDPFA algorithm and MFA algorithm of optimal rate of only $98 \%, 94 \%$ and $97 \%$, $94 \% ;:$ in the high-dimensional function optimization for Griewank function only NFA algorithm and MFA algorithm can find the optimal solution, and the NFA algorithm optimization rate reached $100 \%$, the NFA algorithm on the mean and variance values are far better than the other two algorithms; for Rosenrock function, because of its difficult to determine the optimal direction, so it is difficult to find the optimal solution, but the NFA algorithm to find the solution of more is better than the other two; for Sphere function NFA algorithm can quickly find the optimal solution, while the other two algorithms fail to find the optimal value; it can be seen from the variance values on both optimization of low dimensional function and high dimensional function when the NFA algorithm is more stable than the other two; on the average run time NFA algorithm is much better than the other two algorithms. It shows that the NFA algorithm has better robustness and stability and rapidity operation.

\section{CONCLUSION}

This paper proposed a normalization firefly algorithm based on the improved learning mechanism according to the easily premature convergence and other defects when optimizing the functions with the basic FA. In the algorithm, the position initialization is carried out on the firefly population by virtue of the chaos sequence, thereby, increasing the diversity of the global search; and then, by means of the adaptive adjustment of the dynamic distance weight and step size, the algorithm is capable of carrying out global search from local search, thereby, increasing the information exchange and learning between the fireflies, and meanwhile, accelerating the convergence speed, and improving the searching precision. When the algorithm falls into the local optimum, the introduction of the Gaussian mutation ensures the accuracy of the optimization result more. After the contrastive analysis on the experimental result of several test functions, it can be stated that, the performance that NFA proposed in this paper optimizes the multimodal function is improved substantially. Compared with other intelligence algorithms, NFA can find the accurate solution in a shorter time, and have small volatility and strong robustness. The several improvement schemes proposed in this paper have strong applicability that can be used as the basis of the optimization of the swarm intelligence algorithms. In the future research work, the development will be carried out by surrounding the diversity of the algorithm, and the algorithm will be promoted and applied in more actual projects.

\section{ACKNOWLEDGMENT}

The work was supported by Ningbo Natural Science Foundation (No.2014A610069), Scientific Research Fund of Zhejiang Provincial Education Department (No. Y201326770), Zhejiang Xinmiao Talents Project (No. 2015R405031), the Twelfth Five-Year Plan of Zhejiang Province Key Discipline (Computer Application technology), China.

\section{REFERENCES}

[1] YANG Xin-she. Nature-inspired metheuristic algorithms[M]. Luniver Press, 2008: 83-96.

[2] Praveen Ranjan Srivatsava, B.Mallikarjun, Xin-She Yang. Optimal test sequence generation using firefly algorithm Original Research article [J].Swarm and Evolutionary Computation,2013,8(1):44-53.

[3] YANG Xin-she, DEB S. Eagle strategy using levy walk and firefly algorithms for stochastic Optimization[J].Studies in Computational Intelligence 2010,28(4):101-111

[4] K.Chandrasekaran,Sishaj P.Simon. Network and reliability constrained unit commitment problem using binary real coded firefly algorithm [J]. International Journal of Electrical Power \& Energy Systems, 2012, 43(1): 921-932

[5] Ahmad Kazem, Ebrahim Sharifi, Farookh Khadeer Hussain, Morteza Saberi, Omar Khadeer Hussain.Support vector regression with chaos-based firefly algorithm for stock market price forecasting [J].Applied Soft Computing, 2013 ,13(2):947-958.

[6] Leandro dos Santos Coelho, Viviane Cocoa Mariana. Firefly algorithm approach based on chaotic Tinker bell map applied to multivariable PID controller tuning $[\mathrm{J}]$. Computers \& Mathematics with Applications, October 2012, 64(8): 2371-2382; 
[7] Mohammad Kazak Sayadi , Ashkan Hafezalkotob,Seyed Gholamreza Jalali Naini. Firefly-inspired algorithm for discrete optimization problems: An application to manufacturing cell formation [J].Journal of Manufacturing Systems, 2013, 32(1): 78-84

[8] FENG Y, LIU J, HE Y. Chaos-based dynamic population firefly algorithm[J]. Journal of Computer Applications, 2013,33(3):796-799.
[9] A.H.Gandomi X.-S.Yang, S.Talatahari, A.H.Alavi, Firefly algorithm with chaos $[\mathrm{J}]$. Communications in Nonlinear Science and Numerical Simulation, 2013,18(1):89-98

[10] FU Qiang;TONG Nan;ZHAO Yi-ming, Firefly algorithm based on multi-group learning mechanism[J]. Application Research of Computers, 2013,30(12):3600-3603 